\title{
La technologie électronucléaire en France aujourd'hui
}

Henri Safa, physicien, CEA

L'actuel parc nucléaire français est constitué de réacteurs à eau pressurisée. Avant d'être mise en réacteur, la matière combustible, constituée d'uranium, résulte d'un long processus qui démarre à la mine et passe par des phases d'enrichissement et de préparation des crayons combustibles. Après irradiation en réacteur, les combustibles usés sont séparés et conditionnés pour être mis aux déchets. En France, une partie de ces combustibles fait l'objet d'un retraitement qui permet l'utilisation du plutonium, ce qui ajoute des étapes cruciales et stratégiques pour l'ensemble de la filière.

\section{Le combustible nucléailre}

La densité d'énergie du combustible nucléaire

La fission de l'atome d'uranium dégage une quantité d'énergie très importante par unité de masse de combustible, cent mille fois plus que pour la plus concentrée des énergies fossiles. Ainsi, une pastille de quelques grammes d'uranium enrichi peut fournir dans nos réacteurs nucléaires actuels autant d'énergie thermique que cinq barils de pétrole ${ }^{(a)}$ (fig. 1). Cela explique deux avantages du nucléaire : il a recours à de faibles quantités en ressources naturelles, et par là-même est peu dépendant des fluctuations de leur cours.

Cependant, tandis que la combustion à l'air des hydrocarbures est relativement simple, l'usage du nucléaire requiert une technicité et des compétences élaborées. Il faut maitriser les éléments radioactifs tout au long des étapes de la chaine du combustible nucléaire, afin qu'ils ne puissent entrainer aucune conséquence ni sur l'être humain, ni sur l'environnement. Les précautions sont particulièrement nécessaires lorsque le combustible nucléaire est déchargé du réacteur, à cause de la présence d'éléments hautement radioactifs, même s'ils sont produits en faible quantité.

La matière première uranium

L'uranium est un élément chimique dit "lourd ", c'est-à-dire que son noyau est gros. Il est relativement abondant dans la croute terrestre, autant que l'étain ${ }^{(b)}$. On le trouve partout. Certains gisements sont très riches et offrent des teneurs dépassant les 20\%, à l'instar de Cigar Lake au Canada. L'uranium est obtenu dans les mines (fig. 2) en utilisant des techniques d'extraction comparables à celles d'autres métaux, à la différence notable d'une présence de radioactivité due à l'exhalation 


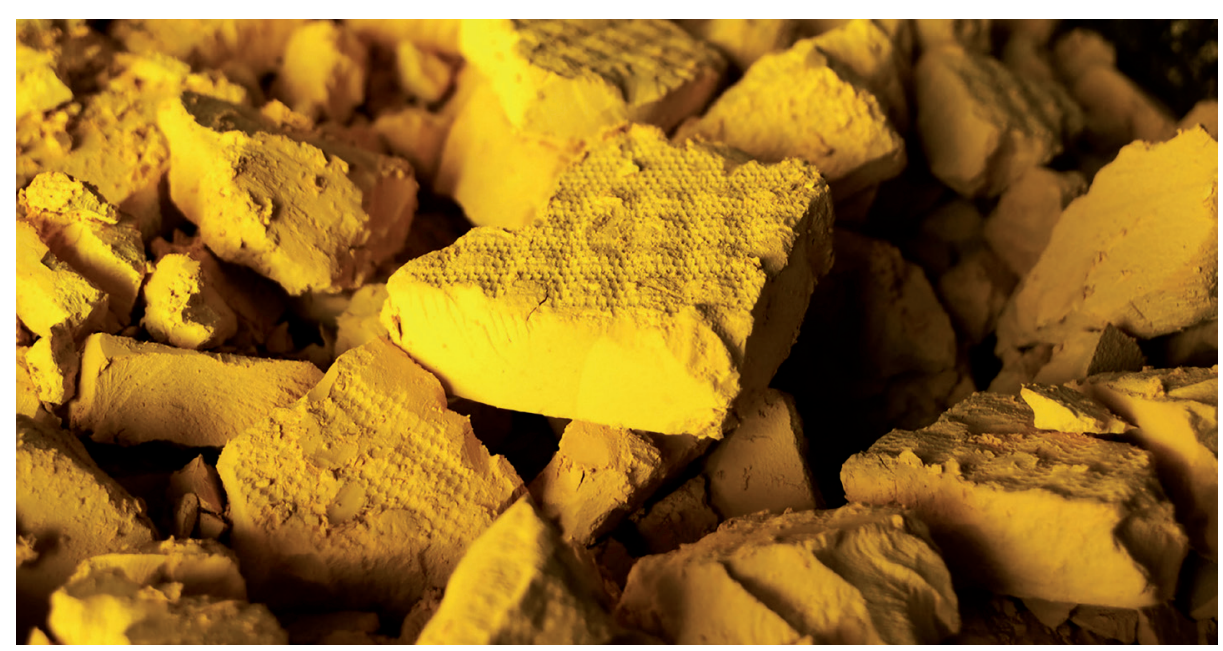

2. Pâte jaune ("yellowcake") obtenue en sortie de mine, après concentration du minerai d'uranium.

du radon dans l'air. La part actuelle du nucléaire dans l'électricité et plus généralement dans l'énergie mondiale étant limitée, l'uranium ne pose pas encore actuellement de problème significatif d'approvisionnement $^{(\mathrm{c})}$. La quantité d'uranium extraite du sol terrestre, typiquement soixante mille tonnes par an, est faible comparativement aux autres minerais ou ressources énergétiques qui se chiffrent habituellement en milliards de tonnes.
En théorie, on pourrait extraire du sol français la totalité du minerai nécessaire pour alimenter annuellement les réacteurs en France ${ }^{(d)}$. On pourrait même l'extraire de l'eau de mer, les limitations pratiques étant le cout économique et énergétique que cela représente. Dans les mines en activité actuellement dans le monde, l'uranium est peu cher (moins de $100 €$ le $\mathrm{kg}$ ), ce qui pèse pour moins de $3 \%$ dans le cout du MWh nucléaire ${ }^{(\mathrm{e})}$. Ainsi,

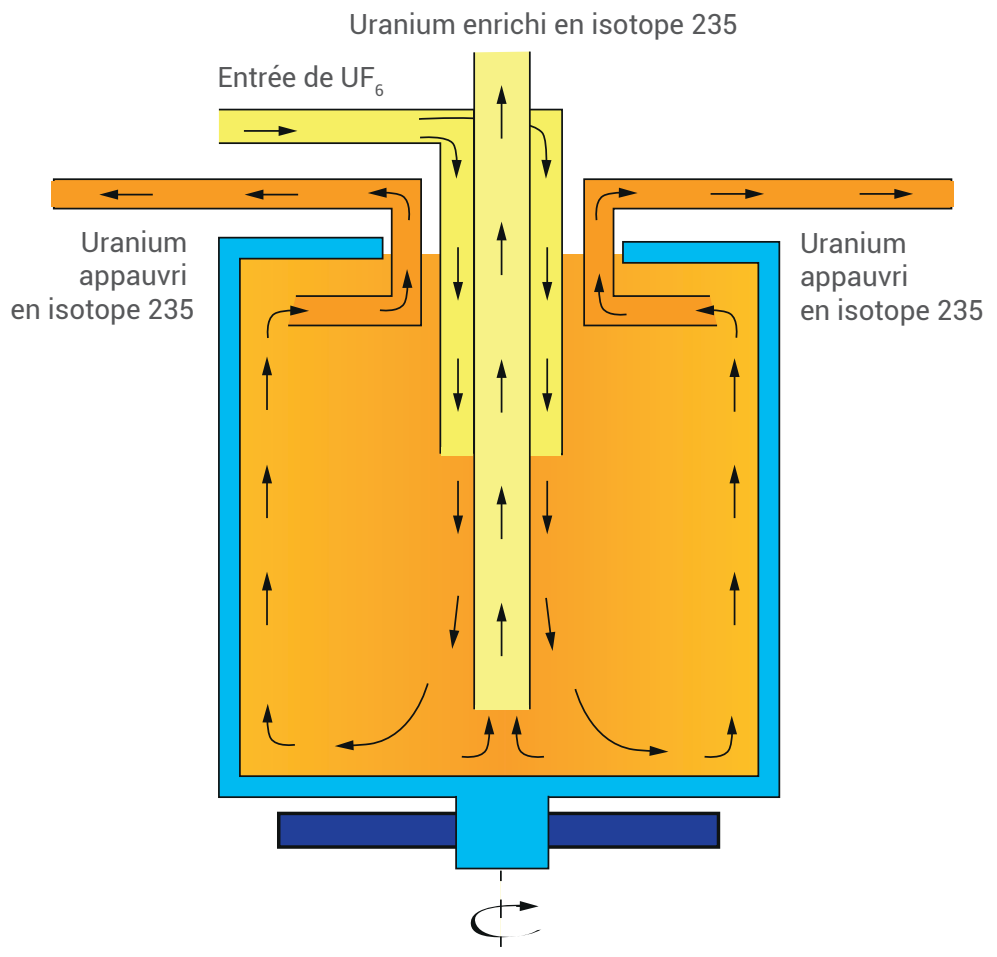

3. Schéma d'une centrifugeuse pour l'enrichissement de l'uranium.

contrairement aux combustibles fossiles, ce qui définit l'indépendance énergétique n'est pas l'accès à la matière première : c'est plutôt l'accès aux technologies spécifiques (réacteurs et usines du parc) qui permettent son exploitation.

\section{L'amont du réacteur (conversion, enrichissement, fabrication du combustible)}

L'uranium naturel se compose de trois isotopes : l'uranium 234, ultra-minoritaire, l'uranium 235, présent naturellement à $0,7 \%$, et l'uranium 238 , très majoritaire ${ }^{(\mathrm{f})}$ Cependant, seul l'isotope ${ }^{235} \mathrm{U}$ est fissile, c'est-à-dire qu'il peut se scinder en deux parties suite à l'absorption par le noyau atomique d'un neutron en libérant de l'énergie. C'est d'ailleurs le seul atome fissile existant sur notre planète ; bien que radioactif, son existence a perduré depuis la formation de la Terre grâce à sa longue demi-vie de 700 millions d'années ${ }^{(\mathrm{g})}$. On dit que l'uranium est " enrichi " quand on augmente sa teneur en atomes fissiles. Il faut atteindre 4\% d'atomes fissiles dans le combustible pour pouvoir maintenir la réaction en chaine dans le cœur d'un réacteur à eau légère.

Pour ce faire, l'uranium doit être d'abord transformé en hexafluorure d'uranium $\left(\mathrm{UF}_{6}\right)$, composé qui possède l'avantage de devenir facilement gazeux : il passe directement de l'état solide à l'état gazeux dès que sa température dépasse $56,4{ }^{\circ} \mathrm{C}$. Cette étape de conversion par fluoration de l'uranium s'effectue dans les usines 


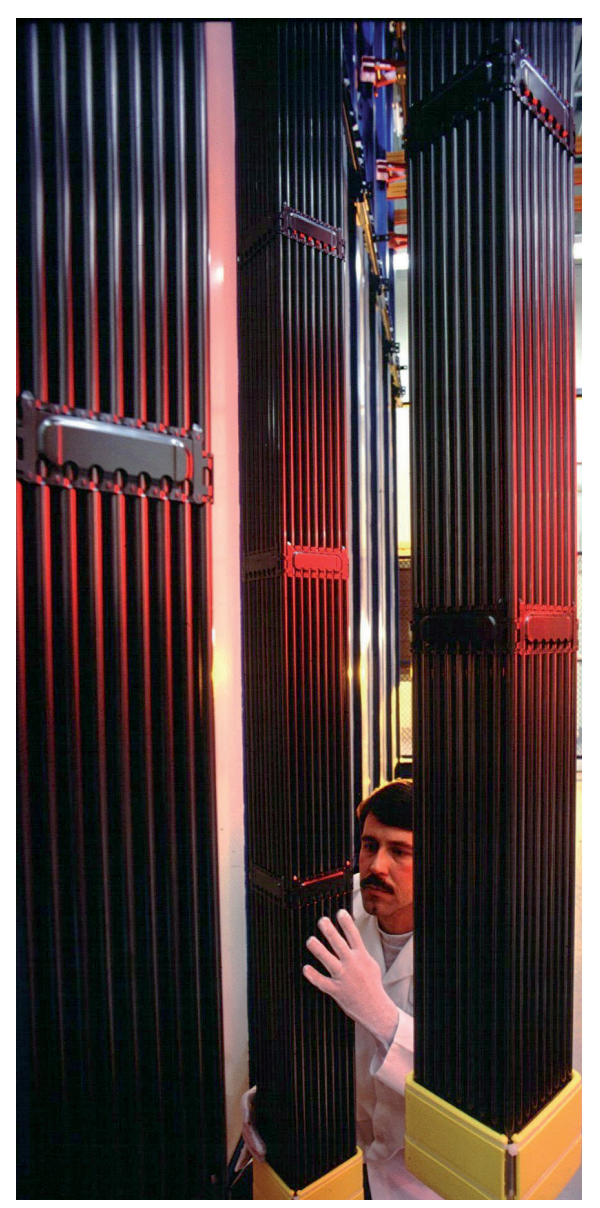

4. Assemblage de combustible nucléaire d'un réacteur à eau pressurisée comprenant une barre de contrôle. Les grilles assurent le maintien mécanique des 264 crayons combustibles en place.

\section{\>}

Comurhex sur les sites de Malvési (Aude) puis du Tricastin (Drôme).

Une fois l'uranium mis sous forme gazeuse d'hexafluorure, on peut procéder à l'étape de l'enrichissement par ultracentrifugation $^{(\mathrm{h})}$. Ce procédé utilise l'action de la force centrifuge agissant sur un gaz contenu dans un récipient tournant à grande vitesse autour d'un axe (fig. 3) L'intensité de la force centrifuge étant proportionnelle à la masse des corps, les atomes d'uranium 238, légèrement plus lourds, sont projetés vers la périphérie. Le gaz au centre du récipient s'enrichit alors en isotope uranium 235, tandis que le gaz près de la paroi est appauvri. Un prélèvement du gaz au centre du récipient fournit un coefficient d'enrichissement suffisant pour qu'une dizaine de centrifugeuses mises en cascade permettent d'atteindre les $4 \%$ souhaités ${ }^{(i)}$. Compte tenu des pertes, il faut typiquement $8 \mathrm{~kg}$ d'uranium naturel pour obtenir $1 \mathrm{~kg}$ d'uranium enrichi.
Une fois enrichi, le gaz est retransformé en poudre solide d'oxyde d'uranium dans un four rotatif avec de la vapeur d'eau à environ $800^{\circ} \mathrm{C}$ (pyrohydrolyse). La poudre est ensuite compactée, concassée puis compressée pour prendre la forme géométrique d'une pastille cylindrique pleine d'environ $1 \mathrm{~cm}$ de diamètre pour $1,3 \mathrm{~cm}$ de hauteur (fig. 1). Un frittage ${ }^{(j)}$ sous hydrogène à $1750^{\circ} \mathrm{C}$ achève la fabrication en garantissant la porosité nécessaire. Ces pastilles sont ensuite insérées les unes au-dessus des autres dans un long tube, une gaine en alliage de zirconium de 4 mètres de long, scellé à ses extrémités, appelé " crayon combustible ", qui contient environ 300 pastilles. Un assemblage comprend 264 crayons combustibles agencés en un carré de 17 par 17 (fig. 4), avec 25 emplacements libres pour guider 24 crayons absorbants assurant le contrôle de la réaction en chaine (et un tube guide pour l'instrumentation). Le cœur d'un réacteur à eau pressurisée (REP) de 1300 MW est composé de 193 assemblages de ce type, le réacteur pressurisé européen (EPR) en construction à Flamanville de 241 assemblages.

\section{Le passage en réacteur}

Un assemblage séjourne environ quatre années dans le cœur du réacteur, temps pendant lequel les noyaux d'uranium 235 fissionnent sous l'action d'un flux neutronique intense produit par la fission nucléaire. En effet, la fission d'un noyau d'uranium produit en moyenne trois neutrons. Ceux-ci induisent la fission d'autres noyaux, et ainsi la réaction diverge exponentiellement : c'est une réaction en chaine.

La chaleur dégagée par les réactions nucléaires est emportée par un fluide dit " caloporteur ", qui circule en circuit fermé : c'est le circuit primaire, dont le fluide devient radioactif. Pour éviter la contamination, ce liquide passe à travers un échangeur de chaleur, appelé "générateur de vapeur ", qui permet de transférer la chaleur à un deuxième liquide : c'est le circuit secondaire. Selon le même principe que dans une centrale thermique classique, grâce à la différence de température entre le générateur de vapeur et la source froide (l'eau d'une rivière, ou l'air dans les tours de refroidissement), ce liquide entraine une turbine qui produit de l'électricité. Au final, comme pour une centrale thermique, environ un tiers de l'énergie de réaction est réellement convertie en électricité ; les deux tiers restant chauffent l'environnement.

Pour la production d'électricité, il faut contrôler la réaction en chaine pour ne pas qu'elle diverge. Pour cela, il faut s'assurer qu'un seul des neutrons produits est en mesure de déclencher une autre fission. Le cœur du réacteur est alors dit "critique ", terme à connotation positive indiquant que son régime de fonctionnement est exactement à la limite souhaitée : ni trop faible, ni trop fort, il peut continuer égal à lui-même tant qu'il subsiste du combustible.

Ce contrôle est assuré grâce à un dosage fin d'éléments absorbants neutroniques : soit du bore dans l'eau du circuit primaire ; soit du cadmium dans les barres de commande ; soit du gadolinium dans le combustible. L'ajustement mécanique des barres de contrôle sert au pilotage du réacteur afin d'atteindre la puissance souhaitée par l'opérateur, tout en s'assurant d'une bonne répartition spatiale de la puissance dans le cœur, et de l'absence d'endroits où la température locale serait trop élevée, entrainant une possible ébullition. Certaines barres restent en position haute, afin que leur chute permette un étouffement de la réaction en chaine en cas d'arrêt d'urgence.

Au fur et à mesure du fonctionnement du réacteur, l'uranium 235 fissile disparait progressivement. Dans le même temps, subissant une irradiation neutronique forte et continue, une faible fraction de l'uranium 238, majoritaire, se transforme par capture neutronique en plutonium 239 , isotope qui possède une haute valeur énergétique puisqu'il est également fissile. Au déchargement du réacteur, le combustible usé ne contient plus que 0,85\% d'uranium 235 alors qu'il comporte désormais plus de $1 \%$ d'atomes de plutonium.

La concentration importante de matières nucléaires dans le cœur d'un réacteur (plus de 100 tonnes de combustible) requiert une gestion du risque spécifique lors de son opération. En effet, le risque le plus couramment envisagé de la filière nucléaire est celui de l'accident majeur sur un réacteur en fonctionnement ${ }^{(\mathrm{k})}$. L'occurrence d'une éventuelle fusion de cœur, bien que conforme aux estimations faites lors de la conception à $10^{-4} / \mathrm{an} /$ réacteur, apparait à l'usage comme non négligeable sur les réacteurs de seconde génération : trois accidents majeurs dans 


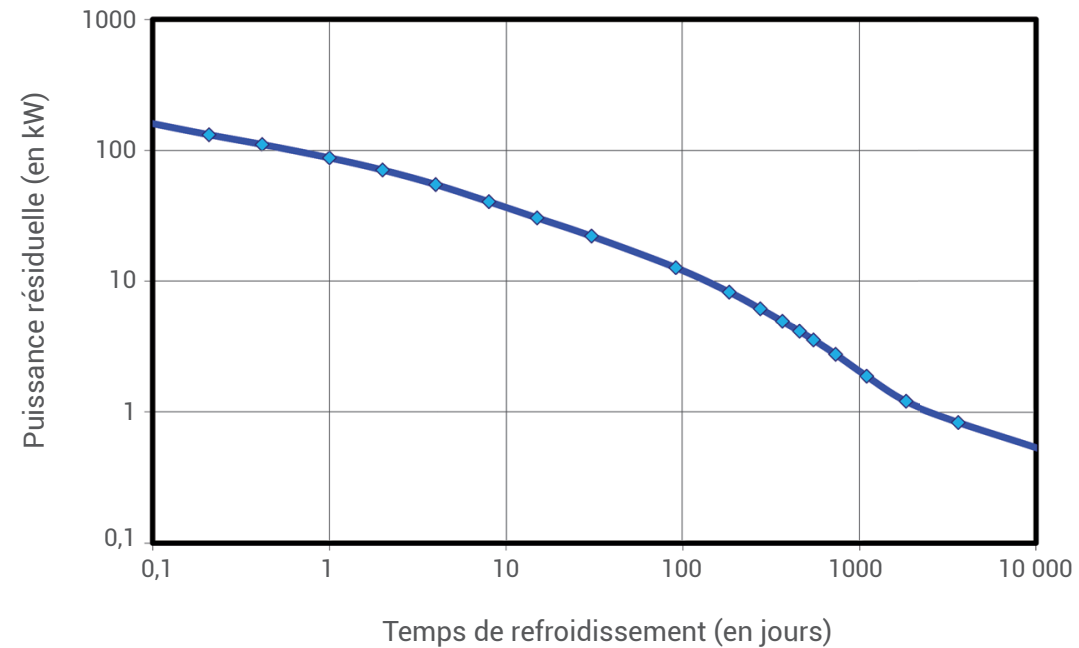

5. Décroissance avec le temps de la chaleur dégagée par les produits de fission et les actinides dans un assemblage combustible (ici un oxyde d'uranium enrichi à $4 \%$ irradié à $45 \mathrm{GWj} / \mathrm{t}$ ).

le monde en 60 ans, dont deux ont causé un relâchement de radionucléides dans l'environnement. Ce qui, dans les années 1970, était considéré par les promoteurs du nucléaire civil comme un accident industriel impossible ou au mieux acceptable, ne l'est plus aujourd'hui.

L'amélioration de la sureté se fait également par l'apprentissage. L'accident de Fukushima nous enseigne que la perte de tout système de refroidissement et celle de l'alimentation électrique externe peuvent dans certaines circonstances survenir simultanément sur une même installation. Auparavant, on se prémunissait contre l'un de ces deux évènements séparément. Aujourd'hui, tous les opérateurs

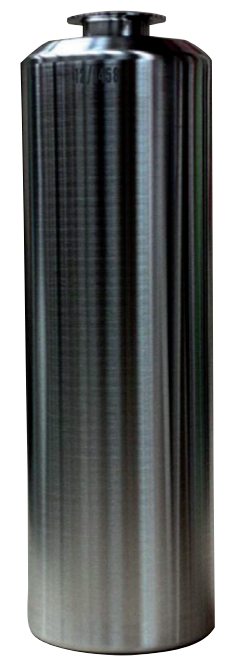

6. Colis de déchets nucléaires. Le conteneur est en acier inox de hauteur 1,35 $\mathrm{m}$ et de diamètre $0,43 \mathrm{~m}$. Il contient environ $400 \mathrm{~kg}$ de verre borosilicaté emprisonnant les déchets. Le colis pèse au total près de 500 kg. dans le monde doivent envisager l'éventualité combinée de ces deux risques.

Aussi, les concepteurs du réacteur pressurisé européen (EPR) se sont-ils focalisés sur l'amélioration de la sureté en réduisant la probabilité de fusion du cœur d'au minimum deux ordres de grandeur, au prix d'un doublement du cout de construction (passant de $1500 € / \mathrm{kW}$ à $3500 € / \mathrm{kW})$. Si tout le parc mondial actuel était constitué de tels réacteurs, on passerait d'une probabilité d'un accident majeur tous les 20 ans à un tous les 2000 ans, voire davantage. Et si par malheur l'accident survient quand même, la radioactivité devrait rester confinée dans l'enceinte quoi qu'il arrive, pour ne pas imposer l'évacuation de la population avoisinante.

\section{Le combustible usé}

La fission nucléaire et l'irradiation neutronique engendrent à l'intérieur du combustible une variété d'éléments radioactifs, appelés "produits de fission ", ayant des demi-vies très différentes. Aussi, lorsqu'un assemblage est déchargé du cœur d'un réacteur, il est fortement radioactif. Par conséquent, même retiré $\mathrm{du}$ cœur du réacteur, le combustible continue d'être chauffé par la puissance résiduelle dégagée par cette radioactivité. Cette chaleur doit être évacuée, sous peine de fusion. L'assemblage est déposé dans une piscine remplie d'eau pour le laisser se refroidir pendant quelques années. Au bout de 2 à 3 ans, la puissance résiduelle de l'assemblage a suffisamment décru (fig. 5) pour qu'il puisse être transporté vers l'usine de La Hague afin d'y être retraité.

Dans l'usine de retraitement, le combustible usé est dissous dans de l'acide nitrique. Les matières nucléaires valorisables comme l'uranium et le plutonium, qui forment l'essentiel du contenu, sont extraites de la solution, tandis que les produits de fission, considérés comme les déchets ultimes de la fission nucléaire, sont vitrifiés et conditionnés dans des colis (fig. 6).

Le retraitement d'environ 70\% du combustible usé en France entraine la fabrication annuelle d'environ 650 colis de haute activité (HA) comprenant des déchets vitrifiés (voir p. 21). Ces déchets vitrifiés renferment $98 \%$ de la radioactivité totale des déchets nucléaires, mais ne représentent que $0,2 \%$ de leur volume total ${ }^{(1)}$. Les déchets de structures (coques, grilles, embouts d'assemblage) sont compactés dans des colis de conteneur similaires et répertoriés comme déchets de moyenne activité à vie longue. Ils représentent quasiment le reste de la radioactivité $(2 \%)$. Tous les autres déchets sont peu radioactifs (moins de $0,03 \%$ de la radioactivité totale).

\section{Le flux des matières nucléaires}

Le parc français est composé de 58 réacteurs à eau pressurisée (REP) (voir dans ce dossier la carte p. 42), alimentés chaque année par environ 1000 tonnes de combustible uranium enrichi à $4 \%$. Le plutonium récupéré dans les combustibles retraités peut être utilisé comme élément fissile en lieu et place de l'uranium 235. Il est alors combiné à de l'uranium appauvri pour constituer le combustible MOX (mélange d'oxydes d'uranium et de plutonium). Depuis quelques années, la totalité des onze tonnes de plutonium produits chaque année sont ainsi intégralement recyclés sous forme de combustible $\mathrm{MOX}^{(\mathrm{m})}$. Quant à l'uranium récupéré à La Hague, dit uranium de retraitement (URT), qui contient encore à peu près autant d'atomes fissiles que l'uranium naturel, il peut donc être enrichi à nouveau pour fabriquer un combustible, l'uranium de retraitement réenrichi (URE), qui alimente les quatre réacteurs de Cruas, en Ardèche ${ }^{(\mathrm{n})}$. Le flux général des matières nucléaires est schématisé sur la figure 7 . 


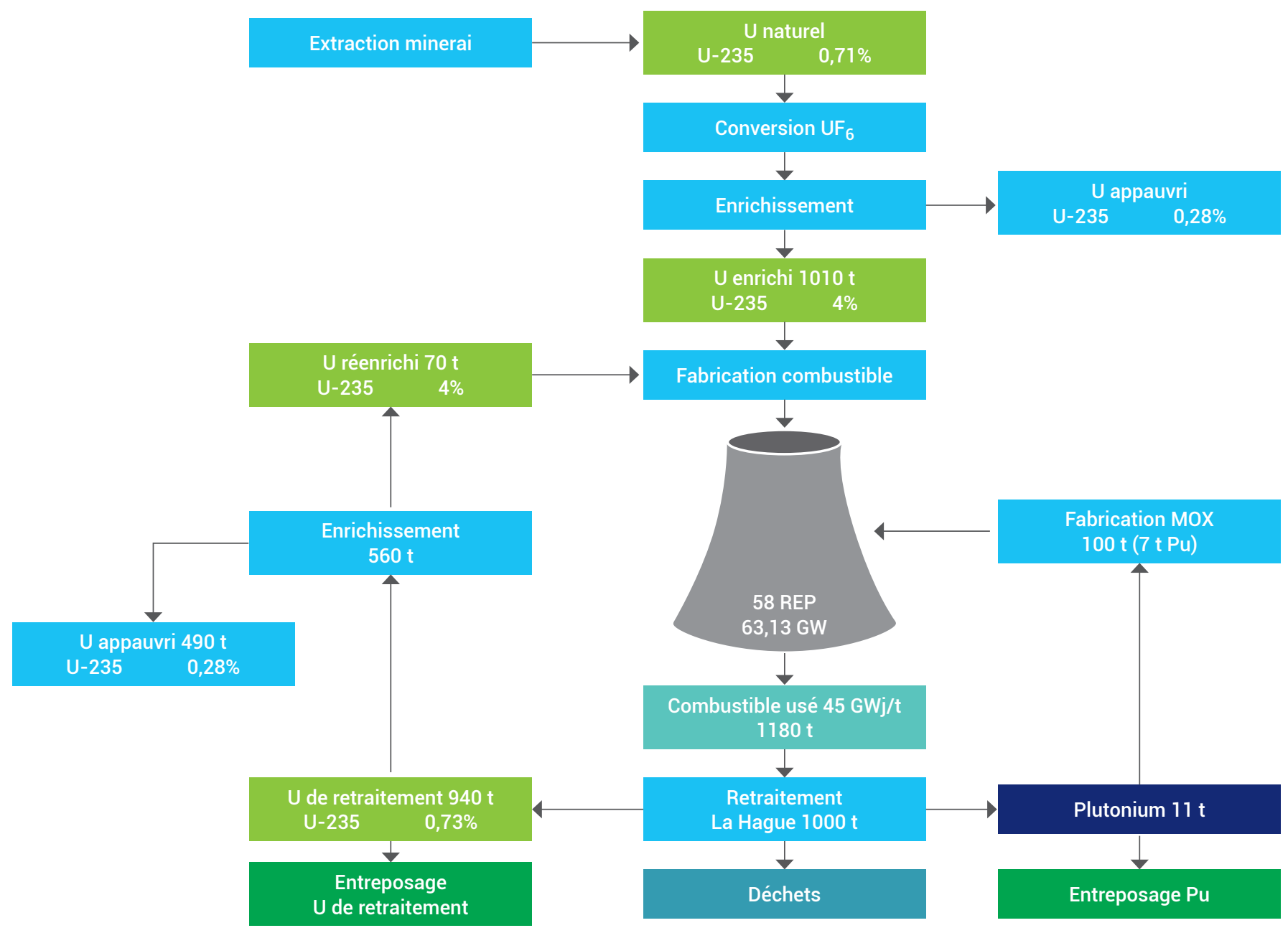

7. Flux annuel des matières nucléaires alimentant l'ensemble du parc français, en prenant comme exemple l'année 2013. En sus des 1000 tonnes de combustible sous forme d'oxyde d'uranium (UOX), la récupération du plutonium permet de fabriquer 100 tonnes de combustibles MOX (mélange d'oxydes d'uranium et de plutonium) et celle de l'uranium 70 tonnes de combustible réenrichi (URE).

a. La densité énergétique du noyau atomique est un milliard de $\mathrm{Wh} / \mathrm{kg}$. Une pastille de $7,5 \mathrm{~g}$ d'uranium enrichi à $4 \%$ peut fournir jusqu'à $9 \mathrm{MWh}$ d'énergie thermique, l'équivalent de 5 barils de pétrole ou d'une tonne de charbon.

b. L'uranium est présent sur Terre à 2 à 3 parties par millions (ppm), avec parfois une concentration supérieure à 1000 ppm dans des zones granitiques ou sédimentaires. C'est 15 fois moins abondant que le cuivre, autant que l'étain (mais pour une production annuelle 5 fois plus faible), 30 fois plus que l'argent, et 600 fois plus que l'or.

c. Pour la question du futur de l'approvisionnement, voir dans ce dossier l'article de S. Bouneau (p. 46).

d. Jusqu'en 2001, l'uranium était encore exploité en France. Cependant, son cout de production n'est pas compétitif sur le marché mondial car les mines françaises sont à faible teneur en uranium (moins de 1\%).

e. Voir dans ce dossier l'article de A.-S. Dessillons (p. 29). Sur les 40 dernières années, le cout du kg d'uranium a fluctué entre 5 et 200 dollars ; en 2018, il est de l'ordre de 40 à 50 dollars par kg.

f. Proportions naturelles : uranium 234 à $0,005 \%$, uranium 235 à $0,711 \%$ et uranium 238 à $99,283 \%$. g. Durée après laquelle la quantitié initiale a été divisée par deux. Il faut attendre au moins une dizaine de demi-vies pour que la radioactivité diminue de façon très significative.

h. Jusqu'en 2011, l'enrichissement était réalisé dans l'usine d'EURODIF à Pierrelatte, par diffusion gazeuse, procédé très énergivore désormais abandonné : trois des quatre réacteurs du Tricastin étaient exclusivement dédiés à l'alimentation électrique de l'usine d'enrichissement. L'ultracentrifugation, nécessitant 40 fois moins d'énergie que la diffusion gazeuse, est aujourd'hui adoptée comme la technologie de référence.

i. Une centrifugeuse enrichit environ 1,2 fois. Donc dix centrifugeuses en cascade enrichissent d'un facteur 1,2 à la puissance 10 , c'est-à-dire 6 fois. La proportion d'uranium 235, initialement de $0,7 \%$, passe ainsi à 6 x $0,7 \%$, c'est-àdire environ $4 \%$.

j. Chauffage de la poudre pour que les grains se soudent entre eux (sans toutefois fondre complètement), comme par exemple lors de la cuisson des poteries.

k. L'amont du cycle, le transport de matières et celui des déchets nucléaires n'ont pour l'instant pas donné lieu à des accidents de très grande ampleur, sauf celui du complexe nucléaire Maïak près de Kychtym (U.R.S.S.) en 1957 dont on sait peu de choses.

1. L'inventaire 2015 de l'Agence nationale pour la gestion des déchets radioactifs (ANDRA) donnait fin 2013 les volumes suivants : $3200 \mathrm{~m}^{3}$ de haute activité (HA) ; $44000 \mathrm{~m}^{3}$ de moyenne activité à vie longue (MAVL);91000 $\mathrm{m}^{3}$ de faible activité à vie longue (FAVL) ; $880000 \mathrm{~m}^{3}$ de faible ou moyenne activité à vie courte (FMAVC) ; $440000 \mathrm{~m}^{3}$ de très faible activité (TFA).

$\mathrm{m}$. Auparavant, une partie du plutonium était réservée pour la fabrication du combustible de réacteurs à neutrons rapides, existants ou à venir.

n. Les évolutions récentes concernent l'augmentation de la production de combustibles MOX (124 tonnes en 2016) et la réduction du combustible URE à 20 tonnes en 2016, compensé par une augmentation du combustible UOX à 1070 tonnes.

\section{En savoir plus}

- Présentation du "Cycle du combustible" français en 2018, Haut Comité pour la transparence et l'information sur la sécurité nucléaire (2018), www.hctisn.fr 